\title{
On the Relationship Between Current and Magnetic Field in Ring-Filaments
}

\author{
Dirk K. Callebaut \\ Physics Dept. UIA, University of Antwerp, B-2610 Antwerp, Belgium
}

\author{
Valentine I. Makarov \\ Pulkovo Observatory, 196140, St. Petersburg, Russia
}

\begin{abstract}
There is a type of filament that forms closed contours encircling regions of one polarity of magnetic field, while the surrounding region has the opposite polarity. One distribution of ring-filaments has sizes $2 \mathrm{R}=40,000$ to $160,000 \mathrm{~km}$ with the maximum around $100,000 \mathrm{~km}$; the other distribution (with filaments and filament channels) has bigger sizes, with a maximum around $300,000 \mathrm{~km}$. At low and mid latitudes the radial component $B_{r s}$ (radial for the Sun) in the region outlined by filaments varies from 50 to 100 gauss, while the longitudinal component $B_{\phi}$ varies from 10 to 30 gauss. The total current inside the filament is $10^{10}-10^{11} \mathrm{~A}$, the ratio $\mathrm{R} / \mathrm{a} \approx 5$ to 10 , the magnetic flux crossing the surface is $\approx 10^{21}-10^{22} \mathrm{Mx}$; the magnetic energy is $\approx 10^{29}-10^{31} \mathrm{erg}$. Polar ring-filaments at latitudes $60^{\circ}-80^{\circ}$ are related to the polar magnetic field reversal and the quasi-flare processes at the poles during the field reversal. Correlation between $B_{r s}$ and $B_{\phi}$ for the polar filament bands cannot be satisfactorily explained. A theoretical model using the conservation laws is used to study the relations between the various fields, currents, etc. of shrinking polar ring-filaments and their evolution. According to this theory ring-filaments should rise higher above the photosphere when shrinking. However, observations show that the height lowers. Presumably the discrepancy is due to the lack of dissipation in the model.
\end{abstract}

\section{Introduction}

The filament and filament channels (weak prominences, dense low chromospheric features, multi-channel systems of matter flow between two nearby prominences) sometimes form a continuous closed contour and outline regions of one polarity. There are two distributions of filament structures: one is a Gaussian distribution (for the sizes from $4-16 \cdot 10^{4} \mathrm{~km}$ with maximum around $10^{5} \mathrm{~km}$ ) - these are pure filament structures; the other one is a Pearson distribution (for the sizes from $10^{5}$ to $6 \cdot 10^{5} \mathrm{~km}$, with maximum around $3 \cdot 10^{5} \mathrm{~km}$ (Makarov et al. 1986)) these consist of pure filaments and filament channels. The structures belonging to the Pearson distribution are formed from those of the Gaussian distribution by merging (the diffusion factor is $\nu \approx 1.5 \cdot 10^{14} \mathrm{~cm}^{2} \mathrm{~s}^{-1}$ ). Most of the structures of the Gaussian distribution are more or less circular ring-filament systems. 


\section{Low-Latitude Ring-Filaments}

Ring-filaments have been detected as closed contours on $\mathrm{H} \alpha$ spectroheliograms at the Kislovodsk Solar Station during 1960-1980 (Makarov and Fatianov 1980) and in the Kodaikanal Observatory plate collection of spectroheliograms in the $\mathrm{H} \alpha$ and CaII-K lines during 1905-1975 (Makarov et al. 1982).

We studied the ring-filament on June 7, 1980 with reference to the magnetic field using Mount Wilson data, Kitt Peak magnetograms and Leroy's data (Leroy 1985 , private comm.). We found that the circular filament outlines an island of " + " polarity with the parameters: big radius $R=50,000 \mathrm{~km}$ and small radius $\mathrm{a}=5,000 \mathrm{~km}$, radial component $B_{r s} \approx 60$ gauss, magnetic field inside filament $B_{\phi} \approx 18$ gauss. It has clockwise orientation and shows inverse polarity. The magnetic flux crossing the surface $\mathrm{S}$ is $F \approx 4 \cdot 10^{21} \mathrm{Mx}$. The total current through $\mathrm{S}$ is $J_{r s} \approx 2 \cdot 10^{11} \mathrm{~A}$. The magnetic energy is $W=L J_{r s}{ }^{2} / 2 c^{2} \approx 2 \cdot 10^{31} \mathrm{erg}$, where $L=2 \pi R \ln (8 R / a-7 / 4)$. See Landau and Lifshitz (1982).

\section{Polar Ring-Filaments}

According to the $\mathrm{H} \alpha$ magnetic charts (1910-1995) one observes ring-filaments at the latitudes $60^{\circ}-80^{\circ}$ of the $\mathrm{N}$ and $\mathrm{S}$ hemispheres, about 2 to 3 years before polar magnetic field reversal. According to Leroy's data (Leroy 1978, 1985, private comm.) the field in polar prominences was oriented from West to East in the North hemisphere before the reversal in the 21st cycle. The field vector and the axis of the filament form a small angle, about $25^{\circ}$. In the South hemisphere the situation was opposite. The field of the prominences is opposite to the background field. Passing from the polar ring-filament to the next neutral line at middle latitude, the longitudinal component of the field, $B_{\phi}$, changes sign and the entire pattern reverses from one cycle to the next. Let us consider the contour created by the polar ring-filament,

$$
\oint \vec{B} \overrightarrow{d l}=\frac{4 \pi}{c} \int \vec{j} \vec{d} S=\frac{4 \pi}{c} J_{r s}
$$

where $d l$ is an element of the contour, $d S$ is an element of the surface surrounded by the contour. In our case $B_{\phi}<0$ and $J_{r s}<0$ (i.e., the current enters the Sun). But the magnetic flux crossing the surface $S$ is positive

$$
\Phi(r)=\int_{s} \vec{B} \overrightarrow{d S}>0
$$

and

$$
\alpha=4 \pi J_{r s} / c F(R)<0
$$

When the radial component of the photospheric magnetic field, $B_{r s}$, in the polar region reverses in the succeeding solar cycle, the longitudinal component, $B_{\phi}$, in filaments reverses as well.

Thus, if $B_{r s}\left(\Theta \geq 60^{\circ}\right)>0$, then $B_{\phi}\left(\Theta \approx 60^{\circ}\right)<0$

and, if $B_{r s}\left(\Theta \geq 60^{\circ}\right)<0$, then $B_{\phi}\left(\Theta \approx 60^{\circ}\right)>0$.

Thus the parameter $\alpha$ does not change. However, at the same time we have

$$
\operatorname{rot} \vec{B}=\alpha \vec{B}
$$


(they are approximately force-free fields) (Makarov and Molodensky 1985). $\alpha(t)$ is such that it never changes its sign; this is connected with the question of stability. We may ask ourselves the following questions:

1. Are there magnetic stars with $\alpha>0$ ?

2. What is the difference between the Sun and those stars?

\section{Model of Ring-Filaments}

The ring-filament is in equilibrium, for certain situations (Figure 1):

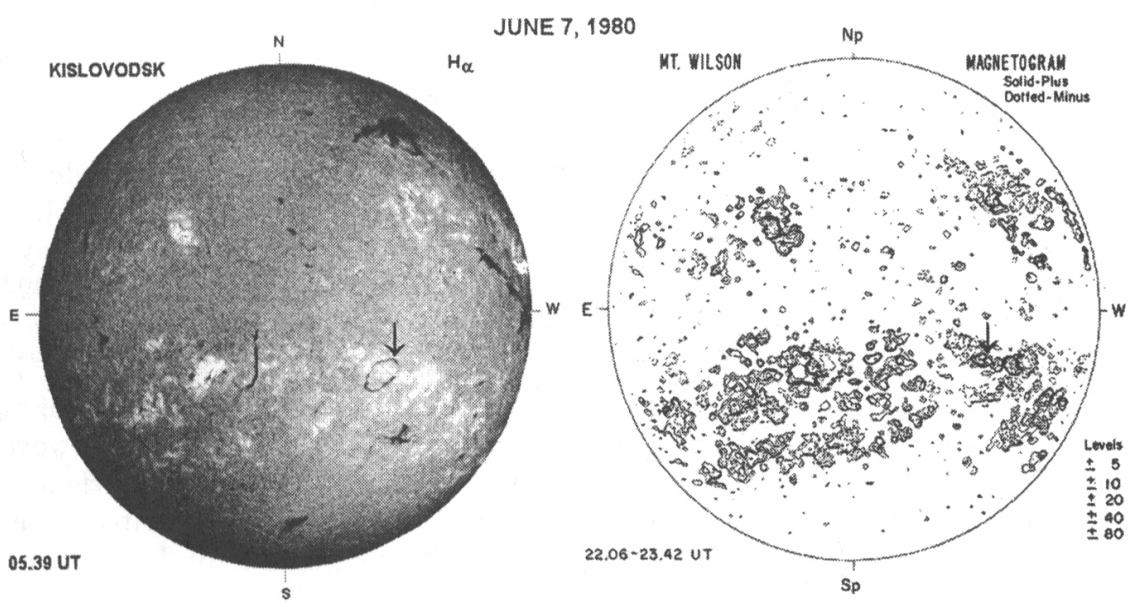

Figure 1.

1. Toroidal and poloidal flux conservation in ring-filament:

$\pi a^{2} B_{\phi} \approx 10^{19} \mathrm{Mx}$.

$\pi a R B_{\Theta} \approx 10^{19} \mathrm{Mx}$, if $B_{\Theta}(r)=r B_{\Theta} / a$ with $r$ the radial coordinate in the torus $(0<r<a)$

We do not use flux conservation of $F(R) \approx \pi R^{2} B_{r s} \approx 10^{21} \mathrm{Mx}$. In fact, as observations show that the field strength inside the ring-filament does not change much when the enclosed surface decreases, it is clear that part of the field and the flux must disappear. How this happens is another matter which is related to the currents, $J_{r s}$ and $J_{\phi}$, (see point 4 below) which suffer losses (due to, e.g., eruptions) which are not taken into account in the present model. But it is clear that flux conservation for $\pi R^{2} B_{r s}$ can not be used straightforwardly.

2. Magnetic energy in ring-filament is proportional to $\mathrm{R}$ :

$a^{2} R\left(B_{\phi}^{2}+B_{\Theta}^{2}\right) \approx 10^{29}-10^{31} \mathrm{erg}$.

3. Mass of ring-filament $a^{2} R \rho \approx 10^{15} \mathrm{~g}$, with $\rho$ the (average) density.

4. From Maxwell's equations:

$J_{r s}=c R B_{\phi} / 2$ and $J_{\phi}=(c / 2) a B_{\Theta} \tan \delta$ with $\delta$ the pitch angle (Figure 1$)$.

5. Usually if $B_{\phi}>B_{\Theta}$, then, according to point 2 above $a^{2} R B_{\phi}^{2} \approx$ constant. Dividing by the mass of the ring-filament (point 3 ) yields $B_{\phi}^{2} / \rho \approx$ const. It may 
be noted here that observations show that $B_{\Theta}$ is rather small after an eruption and then increases gradually. $B_{\phi}^{2} / \rho \approx$ const means that the Alfvén speed along the filament is nearly constant, but it may be higher in the filament channels than in the filaments.

6. We have shown that there is an internal consistency among the various relations indicated here, provided that flux conservation for $F(R)$ is not used.

7. Using the mirror current concept for the vertical force exerted by $J_{\phi}$ is $J_{\phi}^{2} / 2 h c^{2}$, where $\mathrm{h}$ is the height above the solar surface. Equilibrium requires that this equals the total weight of the ring. It follows that $h \approx R^{-2}$, thus the height of the ring-filament increases when the ring-filament shrinks ( $R$ decreasing). However, including losses may allow for a decrease of height.

\section{The Height of the Ring-Filaments at Middle and High Latitudes}

The maximum amount of matter in a prominence is proportional to the density of the magnetic energy of the field and the degree of the curvature of its lines of force. The height of the prominence decreases with a decrease of the energy of the supporting magnetic field. Using the data for cycles 14-19 (Makarov 1994), we reason as follows. If $h^{\prime}$ is a height, $b^{\prime}$ is a base, $\Theta$ is the latitude, then we have from observations $S_{m}\left(\Theta=45^{\circ}\right)=h_{m}^{\prime} \cdot b_{m}^{\prime} \approx 5.9 \cdot 10^{3}$ and $S_{p}\left(\Theta=85^{\circ}\right)=$ $h_{p}^{\prime} \cdot b_{p}^{\prime} \approx 2.0 \cdot 10^{3}$. Usually $b_{p}^{\prime}=0.7 b_{m}^{\prime}$, then $h_{m}^{\prime} / h_{p}^{\prime}=2$. The height of the polar prominences is half the height of middle latitude prominences. However, according to the theoretical model above the height should increase when the ring-filament shrinks. However, in the model we have not yet taken into account dissipation, which probably explains the discrepancy since the total energy and, thus, the current, $J_{l}$, decrease due to dissipation.

Acknowledgments. This work has been done partly under the Collaboration Program Flemish Community-Russia. One of us (V.I.M.) thanks the Russian Foundation For Basic Research, Grant 96-02-16732 for financial support.

\section{References}

Landau, L. and Lifshitz, E. 1982, Electrodynamics of Solid Bodies, Nauka, Moscow

Leroy, J. L. 1978, A\&A, 64, 247

Makarov, V. I. 1994, Solar Phys., 150, 359

Makarov, V. I. and Molodensky, M. M. 1985, Soln. Dann. Bull., 5, 78

Makarov, V. I. and Fatianov, M. P. 1980, Soln. Dann. Bull., 10, 96

Makarov, V., Stoyanova, M. and Sivaraman, K. 1982, J. Astrophys. Astr., 3, 379

Makarov, V., Tavastcherna, K. and Sivaraman, K. 1986, Astron. Zhurn. (Russian), 63, 534 